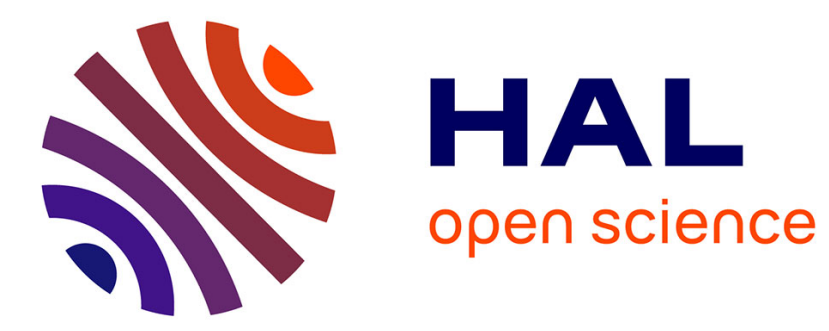

\title{
Label-free immuno-sensors for the fast detection of Listeria in food
}

Alexandra Morlay, Aurore Duquenoy, Félix Piat, Roberto Calemczuk, Thibaut Mercey, Thierry Livache, Yoann Roupioz

\section{- To cite this version:}

Alexandra Morlay, Aurore Duquenoy, Félix Piat, Roberto Calemczuk, Thibaut Mercey, et al.. Label-free immuno-sensors for the fast detection of Listeria in food. Measurement Journal of the International Measurement Confederation (IMEKO), 2017, 98, pp.305 - 310. 10.1016/j.measurement.2016.06.038 . hal-01938240

\section{HAL Id: hal-01938240 \\ https://hal.science/hal-01938240}

Submitted on 24 Nov 2021

HAL is a multi-disciplinary open access archive for the deposit and dissemination of scientific research documents, whether they are published or not. The documents may come from teaching and research institutions in France or abroad, or from public or private research centers.
L'archive ouverte pluridisciplinaire HAL, est destinée au dépôt et à la diffusion de documents scientifiques de niveau recherche, publiés ou non, émanant des établissements d'enseignement et de recherche français ou étrangers, des laboratoires publics ou privés. 


\section{Title}

Label-free immuno-sensors for the fast detection of Listeria in food

\section{Running title}

Fast and label-free detection of Listeria in food

\section{Authors}

Alexandra MORLAY ${ }^{1,2,3,4}$, Aurore DUQUENOY 4 , Félix $\mathrm{PIAT}^{4}$, Roberto CALEMCZUK ${ }^{1,2,3}$, Thibaut MERCEY ${ }^{4}$, Thierry LIVACHE ${ }^{1,2,3}$ and Yoann ROUPIOZ $^{\star 1,2,3}$

\section{Affiliations}

1. Univ. Grenoble Alpes, INAC-SPRAM, F-38000 Grenoble, France

2. CEA, INAC-SPrAM, F-38000 Grenoble, France

3. CNRS, INAC-SPrAM, F-38000 Grenoble, France

4. Prestodiag, 1 mail du Professeur Georges Mathé, F-94800 Villejuif

\section{Corresponding author $\left(^{*}\right)$}

yoann.roupioz@cea.fr, Phone: +334 387898 79, Fax: +334 38785145 


\section{Abstract (149 words)}

Listeria monocytogenes is one of the major foodborne pathogen found in a large set of foodstuff. With the aim of decreasing listeriosis occurrence, the early detection of this pathogen in food products is of tremendous interest. So far, the reference method and all the alternative methods require a pre-enrichment step lasting 24 hours to several days. Then, an aliquot of the enriched sample is collected and characterized according different methods including genetic analysis, or beads immunoagglutination. With the aim of significantly decreasing the overall processing time, we propose an approach involving the detection of alive bacteria during their growing phase via the use of an immuno-chip SPR imaging process. Listeria specific antibodies were also produced and chemically grafted on the biochip before being selected for their ability to distinguish different Listeria strains. Finally, this approach allows the detection of a very low number of Listeria monocytogenes present in foodstuff.

\section{Keywords}

Listeria monocytogenes, Listeria spp., real-time detection, SPRi 


\section{Main text}

\section{INTRODUCTION}

The Center for Disease Control and Prevention (CDC) estimates that every year, in the USA, about 48 millions people get sick from foodborne diseases, 128,000 are hospitalized and 3,000 die. Listeria accounts for one of the five most frequent pathogen involved in death due to foodborne illnesses

(source

CDC,

http://www.cdc.gov/foodborneburden/2011-foodborne-estimates.html, accessed on June 27, 2016). In the course of the 2009-2013 period, the number of human listeriosis increased steadily in Europe (1). In 2013, 1,763 cases were reported by the 28 states members of the European Union and lead to 191 deaths. Such trends highlight the interest in food safety monitoring, and particularly to track any Listeria monocytogenes contamination. The Listeria genus gathers seventeen species classified in 4 clades. The first clade includes L. monocytogenes, L. innocua, L. welshimeri, L. seeligeri, L. ivanovii and L. marthii. Another clade contains $L$. fleischmannii, L. aquatica sp. nov. and L. floridensis sp. nov. A third clade consists of L. rocourtiae, L. booriae, L. newyorkensis, L. weihenstephanensis, L. cornellensis sp. nov., L. grandensis sp. nov. and $L$. riparia sp. nov. The last clade contains only Listeria grayi $(2,3)$. Only Listeria monocytogenes and L. ivanovii and L. innocua to a lesser extent have been described as mammalian pathogens, while L. monocytogenes is the most frequent species involved in human listeriosis. This pathogen is a Gram-positive bacteria, facultative anaerobic and able to grow in a wide range of physico-chemical conditions (4): L. monocytogenes preferentially grows at $\mathrm{pH}$ values from 6 to $8(5,6)$, and in a wide range of temperatures from $-1.5^{\circ} \mathrm{C}$ to $45^{\circ} \mathrm{C}(5$, 6 ). This latter characteristic partly explains why L. monocytogenes is 
searched in a large range of refrigerated food samples where the pathogen can still grow. A study led from 2008 to 2010 in Estonia showed the prevalence of $L$. monocytogenes in various food categories. An average of $2.6 \%$ of the tested food products were found contaminated by the bacteria, with the highest levels of contamination in raw meat products $(18.7 \%)$, raw mixed salads (18.5\%) and raw milk $(18.1 \%)$ compare to raw fish $(8.8 \%)(7)$. L. monocytogenes mobility is also strongly affected by the temperature of incubation as flagellin -a protein ensuring the bacterial mobility- is expressed at much higher levels at $20-25^{\circ} \mathrm{C}$ than at $37^{\circ} \mathrm{C}$ (4). Since its first characterization in 1926 (8), L. monocytogenes has been classified into 4 different lineages based on phylogenetic elements. So far, 16 serotypes have been identified for the Listeria genus $(9,10)$, although serovars $1 / 2 a, 1 / 2 b, 4 b$ and $1 / 2 c$ are involved in $95 \%$ of the isolates found in contaminated food or clinical samples (11). Several approaches can be used for the L. monocytogenes detection (12): standard culture based methods with selective and chromogenic media; Immunoassays like Enzyme-Linked Immunosorbent Assay (ELISA), Enzyme-Linked Fluorescent Assay (ELFA) or lateral-flow assays; or molecular methods based on specific genetic sequence amplification and detection by Polymerase-Chain Reaction (PCR) for instance. These detection methods often required one or two preceding steps lasting up to 3 days: a non- (or half-) selective enrichment step allowing the increase of the number of viable targeted micro-organisms present in the sample, followed by a selective enrichment step maintaining background flora at low levels (5). These pre-enrichment steps enable the bacterial concentration to reach the detection limit of the method. To our knowledge, only one example of detection of this pathogenic strain during enrichment has been described so far by incorporating antibody-functionalized nanoparticles (13). In this 
latter work, an immunoassay was developed to aggregate gold and magnetic nanoparticles, thus enabling the pathogen detection by Surface Enhanced Raman Scattering (SERS). This approach, although requiring the addition of nanoparticles in each run, was successful for the

detection of L. monocytogenes contaminating food samples during the enrichment step, therefore significantly reducing both time-to-result and handling time. With the aim of going one step further in the development of easy-to-operate techniques for the specific detection of L. monocytogenes, we designed a specific label-free immunoassay for its detection. This approach lies on the use of SPR imaging of the bacterial growth $(14,15)$. Based on this optical method, we have designed antibody microarrays dedicated to the detection of Listeria spp. either in isolated pure culture but also during the enrichment of food sample. This real-time assay devoted to the detection of Listeria showed remarkable performances in terms of sensitivity and specificity.

\section{MATERIALS \& METHODS}

\section{Bacterial strains}

The Listeria strains used in this study are classified as Lim for Listeria monocytogenes strains and Lis for Listeria other than monocytogenes strains. The ones numbered from $\operatorname{Lim} 1$ to $\operatorname{Lim} 8, \operatorname{Lim} 9$ to $\operatorname{Lim} 15, \operatorname{Lim} 16$ to Lim18, Lim19 to Lim24, Lim25 to Lim28, Lis1 to Lis12 respectively belong to the molecular serotype IIa, Ilb, Ilc, IVb, La and Lb. All of the strains come from naturally contaminated food products (smoked salmon, raw milk, duck leg, etc.) either obtained from Institut Scientifique d'Hygiène et d'Analyse (ISHA, Massy, France) for Lim1-4, Lim9-10, Lim16, Lim19, Lim25 and Lis1-3 or from laboratory ADRIA Développement (Quimper, France) for the remaining strains. Other nonspecific Gram positive and Gram negative strains were used during this 
study. All the Gram negative strains come from ISHA and the Gram positive strains come from ADRIA. The bacterial strains were streaked on Tryptone-Soy Agar (TSA, Biokar diagnostics, Beauvais, France) plates and incubated overnight at $37 \pm 1^{\circ} \mathrm{C}$. Prior to any experiment, one colony of each strain was inoculated in $10 \mathrm{~mL}$ of Brain Heart Infusion (BHI, Biokar diagnostics, Beauvais, France) at $37 \pm 1^{\circ} \mathrm{C}$ during $18 \mathrm{~h} \pm 2 \mathrm{~h}$. The bacterial concentration was then adjusted to 1 MacFarland (McF) with a densitometer (Grant Instruments) using Buffer Peptone Water (BPW, Biokar diagnostics, Beauvais, France). To control the concentration, six serial dilutions (1:10) were carried out with Tryptone Salt (TS, Merck, Darmstadt, Germany) and $100 \mu \mathrm{L}$ of the sixth dilution were spread on two TSA plates. The colonies were counted after overnight incubation at $37 \pm 1^{\circ} \mathrm{C}$.

\section{Food sample preparation}

The samples were prepared by weighting $25 \mathrm{~g}$ of food matrices in a Stomacher® bag (VWR, Radnor PA, USA). Then $225 \mathrm{~mL}$ of Fraser $1 / 2$ were added (Biokar diagnostics, Beauvais, France). Bacterial suspensions of Lim1, $\operatorname{Lim} 10$ and $\operatorname{Lim} 19$ were previously prepared as described above. Then $100 \mu \mathrm{L}$ of the sixth dilution were injected into the bag to inoculate around $10 \mathrm{CFU}$ of Listeria per bag. Bags were blended using a peristaltic blender (bioMérieux, Marcy-l'Etoile, France) and placed into an incubator at $37^{\circ} \mathrm{C}$ for $24 \mathrm{~h} \pm 1 \mathrm{~h}$. The same procedure as described above was applied to assess inocula. A negative sample was also prepared with $25 \mathrm{~g}$ of lettuce and Fraser $1 / 2$ and incubated in the 
same conditions. Presence of Listeria was confirmed by streaking each sample on Compass Listeria (Biokar diagnostics, Beauvais, France). Chromogenic agars were incubated for $24 \pm 1 \mathrm{~h}$ at $37 \pm 1^{\circ} \mathrm{C}$ before control.

\section{Production of antibodies}

For the specific detection of Listeria, we have developed seven polyclonal antibodies (Ab) Ab1, Ab2, Ab3, Ab4, Ab6, Ab7 and Ab8. A chemical treatment with formaldehyde $36.5-38 \%$ (Sigma Aldrich, Saint Louis, USA) was used to inactivate twelve bacterial strains (Lim1-4, Lim9-10, Lim16, Lim19, Lim 25 and Lis1-3). The pellet of a centrifuged overnight culture in BHI, was re-suspended in Phosphate Buffer Saline (PBS, Sigma Aldrich, Missouri, USA) and adjusted to $4 \pm 0.2 \mathrm{McF}$. Following a $0.5 \%(\mathrm{~V}: \mathrm{V})$ addition of formaldehyde, the solutions were incubated $2 \mathrm{~h}$ at $37 \pm 1^{\circ} \mathrm{C}$ under stirring (80 rpm), centrifuged and the pellet were re-suspended in PBS. The chemical treatment efficiency was controlled by spreading $100 \mu \mathrm{L}$ of each solution into TSA plates and in $10 \mathrm{~mL}$ of $\mathrm{BHI}$. The control was performed after an incubation of $48 \mathrm{~h}$ at $37 \pm 1^{\circ} \mathrm{C}$ (no colonies on TSA plates and clear medium expected). Seven New Zealand White rabbits (male) were challenged with $10^{9} \mathrm{CFU} \cdot \mathrm{mL}^{-1}$ of inactivated mixed bacteria respectively with Lim1-3 for Ab1, Lis1-3 for Ab2, Lim3-Lim4-Lim16 for Ab3, Lim1-Lim9-Lim19 for Ab4, Lim2-Lim10Lim19 for Ab6, Lim2-Lim19-Lim25 for Ab7 and Lim9-Lim10-Lim19 for Ab8. All animals obtained three times one dose of each mix in different conditions (complete Freund's adjuvant (1:1), incomplete Freund's adjuvant (1:1) and without adjuvant) at two weeks intervals. The serums were finally purified by affinity chromatography using protein A columns. Each antibody was then suspended in PBS-sodium citrate $\mathrm{pH}$ 7.0-7.4 
and stored at $-20^{\circ} \mathrm{C}$ (Abliance, Compiegne, France). Ab5 (monoclonal antibody kindly provided by CEA, Marcoule, France) was used as a negative control. This immunoglobulin is raised against a irrelevant protein and thus does not react with any bacterial target.

\section{Antibody modification}

The current preparation for the antibodies involves the coupling of antibodies to pyrrole monomers, N-hydroxysuccinimide-pyrrole (NHSpyrrole, ERAS labo, Saint-Nazaire-Les-Eymes, France) (14). After being washed with a PBS solution and concentrated using Vivaspin concentrators (Vivaspin500, 30 kDa cut-off, Sartorius stedim biotech, Göttingen, Germany), antibodies were coupled to NHS-pyrrole (1:1) during $2 \mathrm{~h}$ at room temperature. A filtration was performed using a Vivaspin concentrator at $4^{\circ} \mathrm{C}$ for $5 \mathrm{~min}$ at $15,000 \mathrm{~g}$ to take out the unreacted NHS-pyrrole. The antibodies concentration was measured by UV absorbance $(280 \mathrm{~nm})$ using a Nanodrop ${ }^{\mathrm{TM}}$ spectrophotometer. Antibodies of interest were then diluted with free pyrrole $(20 \mathrm{mM})$ and a microarraying buffer to reach a $1 \mu \mathrm{M}$ final concentration. They were stored at $-20^{\circ} \mathrm{C}$ before surface arraying.

\section{Surface coating onto PlasmoChip ${ }^{\mathrm{TM}}$}

The PlasmoChip ${ }^{T M}$ is a specific biochip covered with a $50 \mathrm{~nm}$-thick gold layer functionalized with antibodies (Prestodiag, Villejuif, France) using a microarray technology (MicroGrid II 600 arrayer, BioRobotics, Cambridge, UK). The antibodies were immobilized on the biochip by initiating an electrochemistry reaction using a platinum wire mounted into a ceramic needle according to Suraniti et al. (16). On the PlasmoChip ${ }^{\mathrm{TM}}$, 6 series of arrayed antibodies were made and each antibody was printed in triplicate. During $15 \mathrm{~min}$, a solution of Bovine Serum Albumin 1\% 
(BSA, Sigma-Aldrich, Saint Quentin Fallavier, France) and PBS is used to passivate the PlasmoChip ${ }^{\mathrm{TM}}$ surface. After repeating this step twice, the surface is washed with distilled water, dried and stored at $4^{\circ} \mathrm{C}$ in a dessicator.

\section{SPR imaging}

The PlasmoChip ${ }^{\mathrm{TM}}$ was mounted into a Prestokit (Prestodiag, Villejuif, France) composed of six separated wells. Before an SPRi analysis, each well was filled with $900 \mu \mathrm{L}$ of Tryptic Soy Broth (TSB, Biokar diagnostics, Beauvais, France) and inserted into a SPRi optical reader (MonoPresto $^{\mathrm{TM}}$, Prestodiag, Villejuif, France), thermalized at $37 \pm 1^{\circ} \mathrm{C}$. Then $100 \mu \mathrm{L}$ of the sample to be tested (1 McF adjusted bacterial solution or food sample) were loaded on the chip and the analysis was launched. The optical reader performs the signal measurements and the software analyzes this signal. At the beginning of the analysis, the software Bacterics (version 1.1, Prestodiag, Villejuif, France) was used to set the regions of interest $(\mathrm{ROI})$ corresponding to spots of antibodies arrayed on the biochip. Images are recorded and the grey levels of pixels belonging to a region of interest are averaged and plotted in real-time. At the end of the analysis, a specific algorithm indicates presence or absence of Listeria spp. in each well, depending on the presence of bacteria of interest.

\section{RESULTS AND DISCUSSION}

\section{Antibody production and biochip design}

In order to increase the specificity of the immunoassay, dedicated antibodies specific to L. monocytogenes were raised against the whole bacteria and purified. Rabbits were then inoculated with different 
combinations of pathogenic bacteria collected from naturally contaminated samples. To that end, several sets of L. monocytogenes strains were prepared to combine different strains having different serotypes (Table 1a). The serotypes were chosen on the basis of the most frequently encountered serotypes to ensure an antibody production matching the widest panel of contaminated samples (4), and for each of them, several L. monocytogenes strains were gathered to increase antigen diversity in each set (Table $1 \mathrm{~b}$ ). Simultaneously, another series of rabbits were inoculated with a mixture of Listeria strains, other than L. monocytogenes, to evaluate the production of polyclonal antibodies specific to other Listeria species.

Following serum extraction and purification, antibodies were conjugated to a pyrrole moiety and electrochemically arrayed (16) on a gold covered SPR biochip (figure 1a). Each antibody was deposited in triplicate at noncontiguous positions on the array (figure 1b) to assess any bias due to either inter-feature variation or inhomogeneous effects due to reactor filling with the sample. Six identical series were deposited on the same biochip, thus enabling the simultaneous analysis of six different samples on a single device.

Bacterial solutions or spiked food samples were loaded on the biochip and cultured at $37 \pm 1^{\circ} \mathrm{C}$ to enable bacterial division. Then the detection of viable bacteria was carried out during the enrichment phase led on the biochip (Figure 2a). The SPR signal shifts observed for each antibody spot on the surface were monitored in-real time in a label-free manner. Figure $2 \mathrm{~b}$ shows a low variability between the three spots of a same antibody. The responses for each triplicate were thus averaged and plotted along with signals corresponding to the negative control (i.e. Ab5 antibody targeting non-bacterial antigens). 
Screening of L. monocytogenes and other bacterial strains with the antibody microarray

With the aim of testing our approach with a large panel of different Listeria strains, more than 180 experiments have been performed on Listeria pure cultures, and 34 experiments were performed with 16 strains other than Listeria spp. (both Gram positive and negative bacteria). For each experiment, controlled levels of bacteria $\left(10^{7}\right.$ CFU. $\mathrm{mL}^{-1}$ in each analysis well) were used and SPR responses of every arrayed antibody were followed and processed to identify positive responses (table 2). 148 Listeria pure cultures with Ila, IIb, Ilc, IVb and La serotypes were analyzed and showed different recognition efficiencies depending on the antibody grafted on the chip. Table 2 summarizes the assays and presents the percentages of positive/negative responses of each antibody for every bacterial strain. Interestingly, a wide panel of efficiency levels was observed depending on the polyclonal antibody arrayed on the biochip. The comparison of performances, for each polyclonal antibody, against characterized immunogenic serotypes (serotypes used for rabbit's inoculation) highlights very specific responses for several strains, but no systematic recognition of the expected target serotype: for instance, Ab6 - produced after inoculation of Ila, IIb and IVb serotypes, recognized $100 \%$ samples spiked with either Ila or Ilc serotypes L. monocytogenes, as well as IIb and IVb serotypes with very high levels (98\% and 92\% respectively). Ab4, corresponding to an immunisation with the same serovars used to produce Ab6 but with different strains, recognized Ilb, $\mathrm{La}$ and $\mathrm{Lb}$ serotype with an similar rate: $98 \%, 56 \%$ and $6 \%$ respectively. However, the recognition efficiency of IIa, IIc and IVb was lower. This might be explained by the fact that strains belonging to the same serotype (and even the same serovar) do not have systematically the same 
immunogenic capacity. Another explanation would be a differential immune sensitivity to the pooled serotype depending on the host animal. Similarly, Ab2 recognized every Lb serotype Listeria samples (100\%) but showed various efficiencies toward L. monocytogenes (ranging from $0 \%$ to $88 \%$ of positive samples depending on the serotype). Ab7 and Ab8 showed highest binding levels toward some of their expected target serotypes (56\% with IVb and $64 \%$ with IVb respectively), but also lower levels with other expected targeted strains (29\% with Ila and $36 \%$ with Ilb). Unexpectedly, Ab1 and Ab3 showed relatively intermediate performances (27-78\%) toward the serotypes they were raised against (Ila and Ila -llc respectively) although higher levels were observed toward non-inoculated serotypes (e.g. for Ab1 60\%). Such results are compatible with the production of polyclonal antibodies targeting other L. monocytogenes antigens than the ones used for serotyping classification. Thanks to the microarray format where several polyclonal antibodies can be simultaneously tested, such a biochip enables the detection of every screened L. monocytogenes serotypes contaminating samples with high efficiencies by at least one antibody (ranging from 88 to $100 \%$ of the assayed samples depending on the serotype). Interestingly no cross-reactivity was observed with other Gram-positive strains, except with Staphyloccocus aureus. This cross-reaction was anticipated as $S$. aureus naturally expresses protein $A$, which binds the Fc domain of any immunoglobulin $G$ with very high affinity. Another evidence strengthening this hypothesis is the lack of reactivity of any antibody with Staphyloccocus epidermidis as this species does not express protein A. This cross-reaction can be detected and corrected thanks to the negative control, Ab5.

Detection of L. monocytogenes in food samples. 
As L. monocytogenes is regularly involved in contamination of raw food (7), experiments have been carried out on lettuce, spiked with three different L. monocytogenes strains (Table 3). The low levels used for food inoculation are compatible with concentrations naturally found. Ab3, Ab4, Ab6, Ab7 and Ab8 recognized the target present in samples either contaminated with Lim1, Lim 9 or Lim 10. Ab2 was not efficient in the detection of Listeria monocytogenes. It is consistent with the production of this polyclonal antibody as it was expected to target other Listeria strains. In a general overview, the detection firstly occurs with Ab6, Ab3 and Ab4. Ab6 was the most efficient antibody as it enabled the detection of Listeria monocytogenes in food matrices in less than 25 hours (24 h of pre-enrichment and $30 \mathrm{~min}$ of analysis). As described above, Ab4, produced after similar immunization to Ab6, but with other strains from the same molecular serotype, has a lower efficiency regarding the detection of Listeria monocytogenes strains. Ab3 allowed, for the three tested strains, a faster detection of the sample contaminated with Lim1 (molecular serotype Ila), which can be linked to the strains used to obtain this antibody. Indeed, among the three strains used for lettuce spiking, two of them belong to the molecular lla serotype. Ab7 and Ab8 were the less efficient ones as they detected the presence of L. monocytogenes after longer incubation times (from one hour and half to three hours). Ab5, directed against a non-bacterial protein, and the absence of any non-specific reaction with lettuce without Listeria confirmed the good specificity of our anti-Listeria antibodies.

\section{CONCLUSIONS}

The standard procedure, based on the ISO 11290-1:2004, requires up to 3 days to detect and enumerate Listeria monocytogenes in food. As described above, few methods appeared since the last years but only 
some of them are usable in food-processing industries with the aim of batch release. The association of PCR and lateral flow immunoassay allowed the detection of less than $10 \mathrm{CFU}$ per $25 \mathrm{~mL}$ of milk of Listeria monocytogenes and other Listeria species within $28 \mathrm{hrs}$ (24 hrs of preenrichment and 4hrs of PCR and analysis) (6, 17). This approach remains complex due to the number of steps and to an important handling time. In the present SPR imaging approach, we have shown how antibody microarrays processed in real-time and in a label-free manner represent a potential easy to use and alternative method allowing the detection of Listeria monocytogenes and other Listeria spp. in less than 25 hours (24h of pre-enrichment step and less than $1 \mathrm{~h}$ of analysis). The developed antibodies possess relatively good performances allowing recognition of $L$. monocytogenes in pure culture and in food sample, especially with Ab6 and Ab4 immunoglobulins. Furthermore, we identify one polyclonal antibody, Ab2, as specific to other Listeria spp species. No cross-reactivity was observed all along the experiments towards any Gram-positive strains or for food background natural flora. Our technology based on the Surface Plasmon Resonance imaging is thus suitable for large-scale routine food safety analysis and gives access to the rapid detection of Listeria at very low initial contamination level, matching with most regulatory requirements.

\section{Acknowledgments}

The work is part of a $\mathrm{PhD}$ thesis (A. M.) funded by the National Association for Research and Technology (ANRT $n^{\circ}$ 624/2013). This project was also partly supported by the Labex ARCANE program (ANR11-LABX-0003-01). 


\section{Conflict of interest}

No conflict of interest to declare. 


\section{References}

1. Eurosurveillance editorial $t$ (2015) The 2013 joint ECDC/EFSA report on trends and sources of zoonoses, zoonotic agents and foodborne outbreaks published. Euro surveillance : bulletin Europeen sur les maladies transmissibles = European communicable disease bulletin 20(4).

2. den Bakker HC, et al. (2014) Listeria floridensis sp. nov., Listeria aquatica sp. nov., Listeria cornellensis sp. nov., Listeria riparia sp. nov. and Listeria grandensis sp. nov., from agricultural and natural environments. International journal of systematic and evolutionary microbiology 64(Pt 6):1882-1889.

3. Orsi RH \& Wiedmann M (2016) Characteristics and distribution of Listeria spp., including Listeria species newly described since 2009. App/ Microbiol Biotechnol 100(12):5273-5287.

4. Farber JM \& Peterkin PI (1991) Listeria monocytogenes, a FoodBorne Pathogen. Microbiological Reviews 55(3):476-511.

5. Magalahaes R, et al. (2014) Listeria monocytogenes. in Encyclopedia of Food Safety, ed Press A (Elsevier, San Diego, CA, USA). 
6. Valimaa AL, Tilsala-Timisjarvi A, \& Virtanen E (2015) Rapid detection and identification methods for Listeria monocytogenes in the food chain - A review. Food Control 55:103-114.

7. Kramarenko T, et al. (2013) Listeria monocytogenes prevalence and serotype diversity in various foods. Food Control 30:24-29.

8. Murray EGD, Webb RA, \& Swann MBR (1926) A disease of rabbits characterized by large mononuclear leucocytosis, caused by a hiterto undescribed bacillus, Bacterium monocytogenes (n. sp.). J. Pathol. Bacteriol. 29:407-439.

9. Orsi $\mathrm{RH}$, den Bakker $\mathrm{HC}$, \& Wiedmann $\mathrm{M}$ (2011) Listeria monocytogenes lineages: Genomics, evolution, ecology, and phenotypic characteristics. International journal of medical microbiology : IJMM $301(2): 79-96$.

10. Jersek B, Tcherneva E, Rijpens N, \& Herman L (1996) Repetitive element sequence-based PCR for species and strain discrimination in the genus Listeria. Letters in applied microbiology 23(1):55-60.

11. Doumith M, Buchrieser C, Glaser P, Jacquet C, \& Martin P (2004) Differentiation of the major Listeria monocytogenes serovars by multiplex PCR. Journal of clinical microbiology 42(8):3819-3822.

12. Jadhav S, Bhave M, \& Palombo EA (2012) Methods used for the detection and subtyping of Listeria monocytogenes. Journal of microbiological methods 88(3):327-341. 
13. Weidemaier $\mathrm{K}$, et al. (2015) Real-time pathogen monitoring during enrichment: a novel nanotechnology-based approach to food safety testing. International journal of food microbiology 198:19-27.

14. Bouguelia S, et al. (2013) On-chip microbial culture for the specific detection of very low levels of bacteria. Lab on a chip 13(20):4024-4032.

15. Mondani L, Roupioz Y, Delannoy S, Fach P, \& Livache T (2014) Simultaneous enrichment and optical detection of low levels of stressed Escherichia coli $\mathrm{O} 157: \mathrm{H} 7$ in food matrices. Journal of applied microbiology 117(2):537-546.

16. Suraniti E, et al. (2007) Real-time detection of lymphocytes binding on an antibody chip using SPR imaging. Lab on a chip 7(9):1206-1208.

17. Kovacevic J, et al. (2009) Evaluation of environmental sampling methods and rapid detection assays for recovery and identification of Listeria spp. from meat processing facilities. J Food Prot 72(4):696-701. 
Figure captions

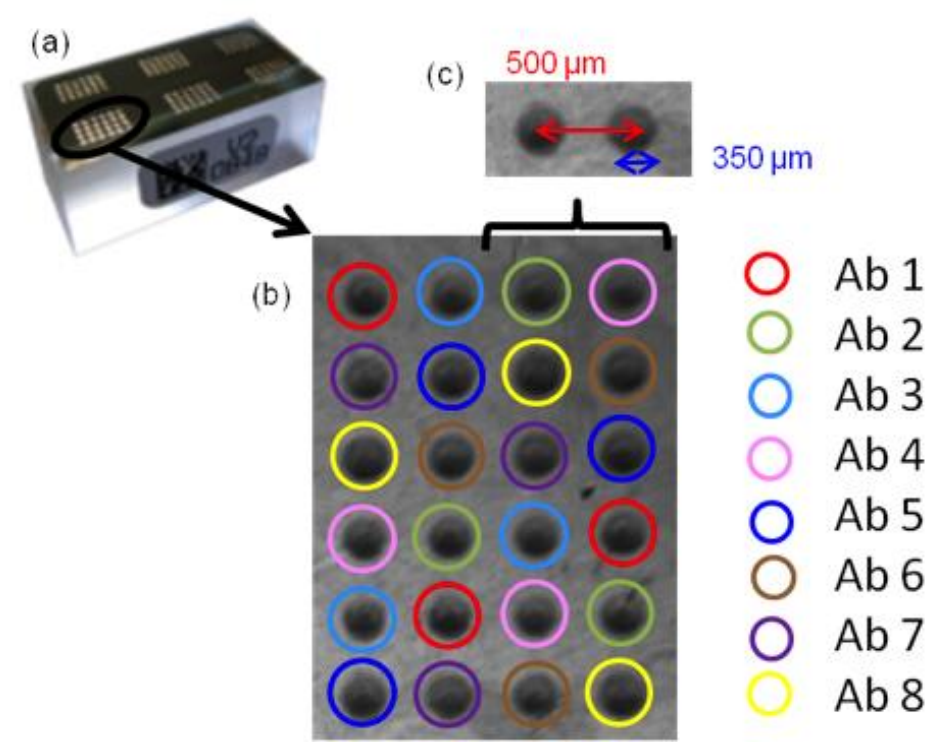

Figure 1: Mapping of the microarrayed antibodies. (a) View of the functionalized PlasmoChip ${ }^{\mathrm{TM}}$ biochip with 6 identical series of antibodies arrayed onto the gold layer. (b) SPR image of one series of proteins containing eight triplicates of antibodies. (c) SPR enlarged image of two features showing the dimension of one spot and the distance between the center of two spots. 

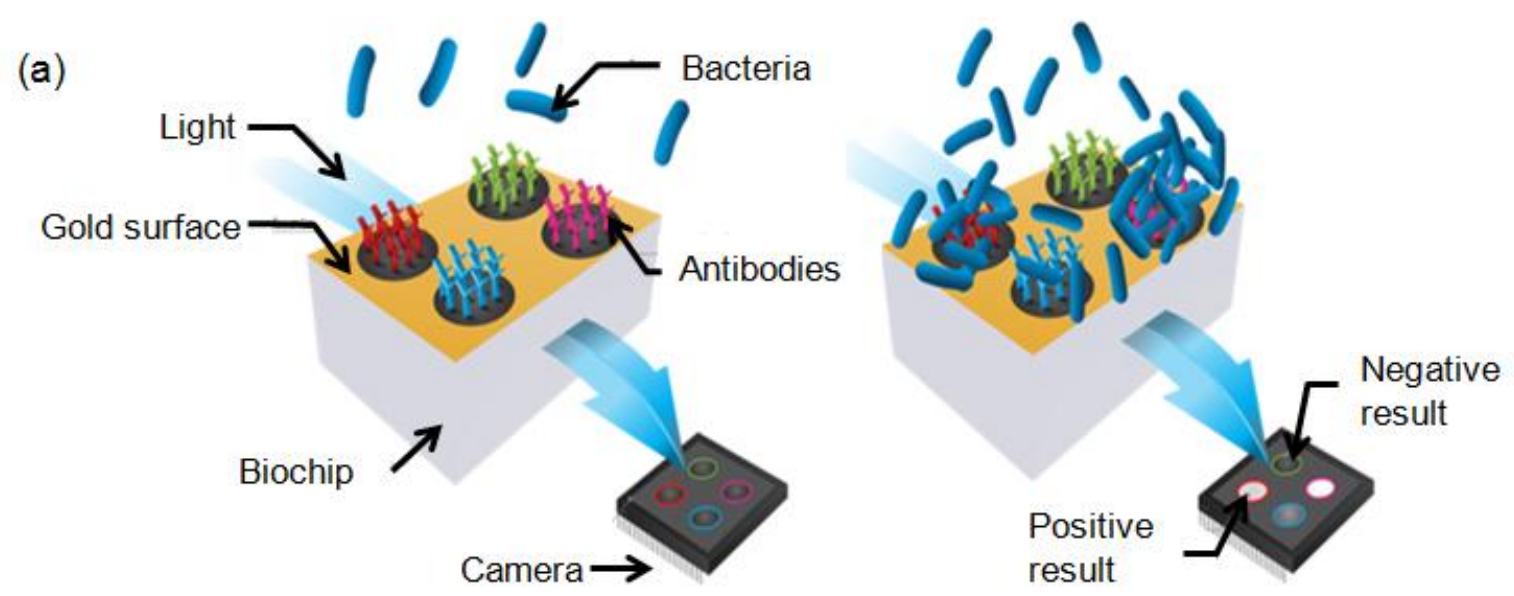

(b)

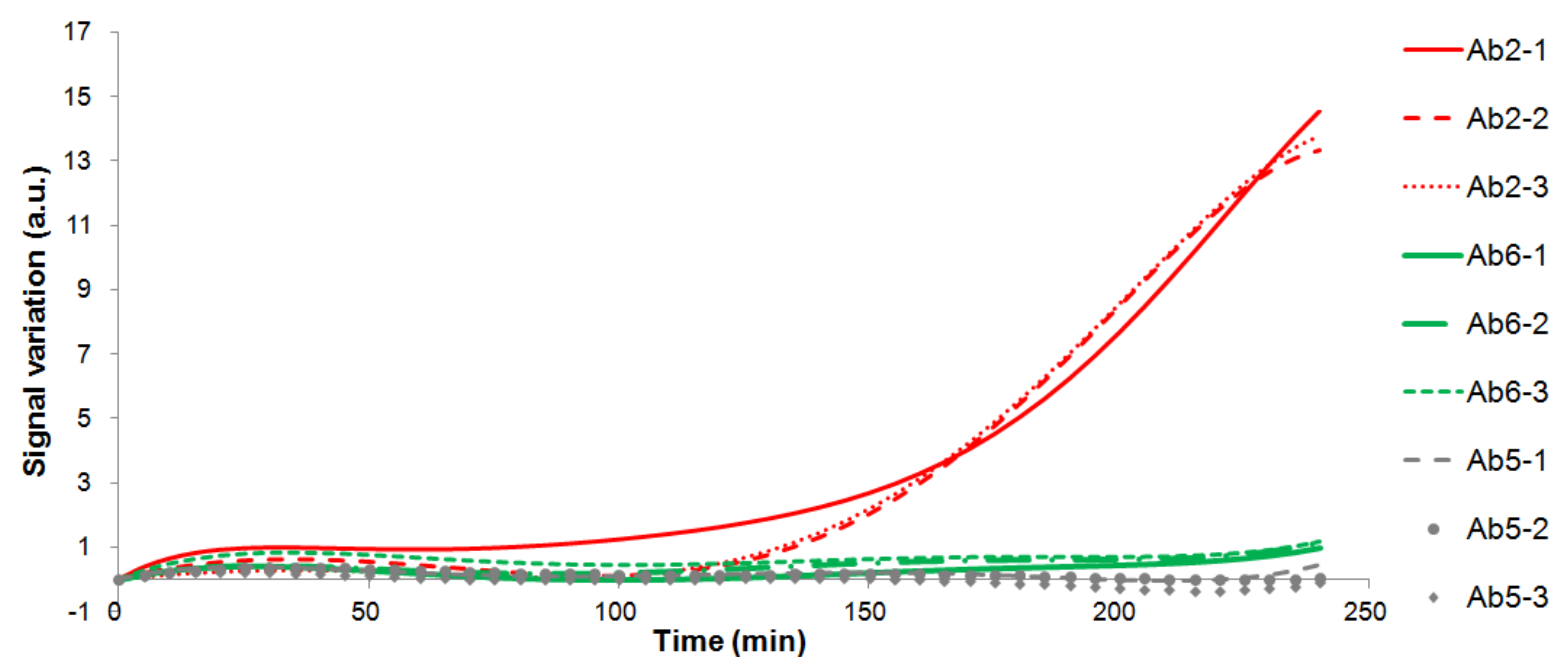

Figure 2: SPR imaging of the biochip and data monitoring in real-time. (a) On-chip culture of bacteria. Viable cells divide on the microarray and the specific binding of bacteria on dedicated antibodies is monitored in a label-free manner. (b) Detection of Listeria welshimeri in pure culture of TSB. Each curve represents SPRi data of one antibody spot: Ab2, which gives a positive result, Ab6, which does not react and the negative control, Ab5. 


\section{Tables}

Table 1: Bacterial strains used for polyclonal antibody production and experiments (NA for non applicable)

Table 2: Bacterial solutions screening on the antibody microarray. Different bacterial strains were tested: L. monocytogenes of Ila, Ilb, Ilc, IVb, La and Lb serotypes, Listeria non L. monocytogenes, 10 other Gram-positive bacterial strains and 6 Gram-negative bacterial strains. Percentages correspond to the ratio of contaminated samples giving a positive response, after 4 hours, on the total numbers of experiments.

Table 3: Processing of contaminated food samples on the antibody microarray. The three bacterial strains Lim1, Lim9 and Lim10 from the molecular serotype IIa, IIb, IVb respectively of L. monocytogenes were tested. The "+" and "-“" signs correspond to positive or negative responses for each antibody. The detection, time expressed in minutes, was determined for each positive response (shaded boxes). 